\title{
The impact of genetic structure on sequencing analysis
}

\author{
Sneha Jadhav ${ }^{1,2}$, Olga A. Vsevolozhskaya ${ }^{2}$, Xiaoran Tong ${ }^{2}$ and Qing Lư ${ }^{2^{*}}$
}

From Genetic Analysis Workshop 19

Vienna, Austria. 24-26 August 2014

\begin{abstract}
Background: Genome-wide association studies have made substantial progress in identifying common variants associated with human diseases. Despite such success, a large portion of heritability remains unexplained. Evolutionary theory and empirical studies suggest that rare mutations could play an important role in human diseases, which motivates comprehensive investigation of rare variants in sequencing studies. To explore the association of rare variants with human diseases, many statistical approaches have been developed with different ways of modeling genetic structure (ie, linkage disequilibrium). Nevertheless, the appropriate strategy to model genetic structure of sequencing data and its effect on association analysis have not been well studied.

Methods: We investigate 3 statistical approaches that use 3 different strategies to model the genetic structure of sequencing data. We proceed by comparing a burden test that assumes independence among sequencing variants, a burden test that considers pairwise linkage disequilibrium (LD), and a functional analysis of variance (FANOVA) test that models genetic data through fitting continuous curves on individuals' genotypes.

Results: Through simulations, we find that FANOVA attains better or comparable performance to the 2 burden tests. Overall, the burden test that considers pairwise LD has comparable performance to the burden test that assumes independence between sequencing variants. However, for 1 gene, where the disease-associated variant is located in an LD block, we find that considering pairwise LD could improve the test's performance.

Conclusions: The structure of sequencing variants is complex in nature and its patterns vary across the whole genome. In certain cases (eg, a disease-susceptibility variant is in an LD block), ignoring the genetic structure in the association analysis could result in suboptimal performance. Through this study, we show that a functional-based method is promising for modeling the underlying genetic structure of sequencing data, which could lead to better performance.
\end{abstract}

\section{Background}

Advancements in sequencing technology have enabled researchers to sequence exome regions or even the whole genome at affordable cost [1]. The emerging sequencing data facilitates the study of massive amounts of single nucleotide variants (SNVs), including both rare and common variants, for their potential role in complex human diseases. Although these studies hold great promise for identification of new disease-susceptibility variants, the extremely large number of SNVs brings

\footnotetext{
* Correspondence: qlu@msu.edu

${ }^{2}$ Department of Epidemiology and Biostatistics, Michigan State University,

East Lansing, MI 48824, USA

Full list of author information is available at the end of the article
}

significant challenge for association analysis. Conventional single-locus analysis suffers from low power because of the low frequency of SNVs and the issue of multiple testing. Grouping SNVs in a genetic region (eg, gene) could aggregate the association signal and alleviate the multiple testing issue, and therefore has been widely used in association analysis of sequencing data [2].

Various statistical methods have been proposed to group SNVs with or without considering the underlying genetic structure (ie, linkage disequilibrium [LD]). However, the impact of different strategies of modeling genetic structure on association results has rarely been investigated. If empirical evidence suggests that use of genetic structure in association analysis does not increase power, it gives us a 
Table 1 Power comparison of 3 tests in the case of unidirectional effects

\begin{tabular}{lllllllll}
\hline Test & \multicolumn{8}{c}{ Proportion of causal variants } \\
\cline { 2 - 9 } & 0.01 & 0.05 & 0.1 & 0.15 & 0.2 & 0.25 & 0.3 & 0.5 \\
\hline BT & 0.343 & 0.617 & 0.714 & 0.766 & 0.759 & 0.776 & 0.793 & 0.781 \\
BTCOV & 0.339 & 0.615 & 0.712 & 0.767 & 0.755 & 0.780 & 0.792 & 0.794 \\
FANOVA & 0.398 & 0.700 & 0.764 & 0.808 & 0.807 & 0.814 & 0.814 & 0.744 \\
\hline
\end{tabular}

basis for excluding this factor from statistical modeling. On the other hand, if it is important to consider LD among SNVs, then we need to investigate appropriate strategies for characterizing the underlying genetic structure. As an initial step to investigate this issue, we chose 3 tests with different ways of modeling LD between SNVs: (a) a weighted burden test assuming independence among SNVs (BT) [3]; (b) a weighted burden test considering pairwise LD (BTCOV) [4]; and (c) a functional analysis of variance (FANOVA) [5] test that considers LD among nearby loci and models the genotype profile of an individual as a continuous function.

\section{Methods}

\section{Burden test}

We consider a burden test developed by Madsen and Browning [3] that assumes independence among SNVs. The test summarizes the genetic score of all SNVs in a genetic region as $\frac{\gamma_{i}=\sum_{j=1}^{L} g_{i j}}{w_{j}}$, where $L$ is the number of SNVs, $g_{i j}$ is the number of low-frequency alleles of the $j^{\text {th }}$ SNV for the $i^{\text {th }}$ individual. The weight is defined to emphasize the effect of rare variants with $w_{j}=\sqrt{n_{j} q_{j}\left(1-q_{j}\right)}$, where $n_{j}$ is the number of controls and $q_{j}$ is the minor allele frequency (MAF) of the $j^{\text {th }} \mathrm{SNV}$ in controls. Analysis of variance (ANOVA) is then used to assess the association between summary genetic scores and the binary phenotype. Because the test simply adds the genotype of each SNV weighted by a function of its MAF, it does not consider LD between SNVs.

Table 2 Power comparison of 3 tests in the case of bidirectional effects

\begin{tabular}{lllllllll}
\hline Test & \multicolumn{8}{c}{ Proportion of causal variants } \\
\cline { 2 - 9 } & .01 & .05 & 0.1 & 0.15 & 0.2 & 0.25 & 0.3 & 0.5 \\
\hline BT & 0.208 & 0.508 & 0.585 & 0.621 & 0.665 & 0.678 & 0.703 & 0.683 \\
BTCOV & 0.200 & 0.509 & 0.579 & 0.618 & 0.663 & 0.668 & 0.698 & 0.680 \\
FANOVA & 0.217 & 0.597 & 0.683 & 0.732 & 0.765 & 0.799 & 0.809 & 0.815 \\
\hline
\end{tabular}

Table 3 Summary of the 10 genes with the smallest $p$ values from the association analysis

\begin{tabular}{llll}
\hline Gene & \multicolumn{3}{c}{ Test } \\
\cline { 2 - 4 } & BT & BTCOV & FANOVA \\
\hline SUMF1 & $8.75 \mathrm{E}-05$ & $8.43 \mathrm{E}-05$ & $1.31 \mathrm{E}-05$ \\
RELB & $6.57 \mathrm{E}-02$ & $7.08 \mathrm{E}-02$ & $4.35 \mathrm{E}-04$ \\
HIF3A & $2.12 \mathrm{E}-02$ & $2.19 \mathrm{E}-02$ & $4.34 \mathrm{E}-03$ \\
THRA & $1.85 \mathrm{E}-02$ & $9.68 \mathrm{E}-03$ & $3.62 \mathrm{E}-02$ \\
TFDP1 & $1.50 \mathrm{E}-02$ & $1.13 \mathrm{E}-02$ & $1.95 \mathrm{E}-02$ \\
PROK2 & $1.23 \mathrm{E}-02$ & $1.32 \mathrm{E}-02$ & $1.27 \mathrm{E}-01$ \\
POLR2A & $2.10 \mathrm{E}-02$ & $2.25 \mathrm{E}-02$ & $1.31 \mathrm{E}-02$ \\
CD1C & $2.76 \mathrm{E}-02$ & $1.42 \mathrm{E}-02$ & $5.27 \mathrm{E}-02$ \\
CCL24 & $2.24 \mathrm{E}-02$ & $2.71 \mathrm{E}-02$ & $1.92 \mathrm{E}-02$ \\
MAP3K6 & $8.72 \mathrm{E}-02$ & $8.45 \mathrm{E}-02$ & $3.27 \mathrm{E}-02$ \\
\hline
\end{tabular}

Burden test that considers pairwise linkage disequilibrium In addition to the above burden test, we also consider another type of burden test proposed by Schaid et al [4], which considers pairwise LD. We consider the following summary of genetic scores, $\frac{S_{i}=\sum_{j=1}^{L} g_{i j}}{w_{j}}$, where $w_{j}$ and $g_{i j}$ are defined in the same manner as BT. However, unlike the conventional burden test, the test statistic of BTCOV is given by $T=\frac{\left((Y-\bar{Y})^{\prime} S\right)^{2}}{(Y-\bar{Y})^{\prime} V_{S}(Y-\bar{Y})}$, where $Y$ denotes the vector of disease status and $\bar{Y}$ is the mean disease status. $V_{s}$ is the covariance matrix, where $v_{i i^{\prime}}=\sum_{j=1}^{L} \sum_{j^{\prime}=1}^{L^{\prime}} \frac{1}{w_{j}} \frac{1}{w_{j}^{\prime}} \operatorname{cov}\left(g_{i j}, g_{i^{\prime} j^{\prime}}\right)$.

\section{Functional analysis of variance}

FANOVA considers LD by fitting a continuous function (curve) on the genotype data of an individual [5]. While various smoothing methods can be used to fit curves on individuals' genotype data, we used cubic B-splines to fit the smooth functions [6]. By using cubic B-splines [5], we first fit $g_{i k}(t)$, the smoothed function of genetic variants at the genomic position $t$ for an individual $i$ in the $k$ th group. The FANOVA can then be used to compare the difference of curves in cases and controls. The FANOVA model can be written as:

$$
\begin{aligned}
& g_{i k}(t)=\mu_{k}(t)+\epsilon_{i k}(t) \\
& \epsilon_{i k}(t) \rightarrow G . P(0, \gamma), \quad i=1,2, \ldots, n_{k}, \quad k=1,2
\end{aligned}
$$

where $i, k, n_{k}$ and $t$ denote the individual, the group (ie, case or control), the total number of individuals in the $k^{\text {th }}$ group, and the genomic position of a genetic variant, respectively. G. $P(0, \gamma)$ stands for the gaussian process, where $\gamma$ is the covariance function, ${ }_{i k}$ is the 
error term and $\mu_{k}$ is the mean function for the $k^{\text {th }}$ group. We test the following hypotheses:

$$
H_{0}: \mu_{1}(t)=\mu_{2}(t) \forall t H_{1}: \mu_{1}(t) \neq \mu_{2}(t)
$$

for some $t$.

Similar to ANOVA, the test statistic for the hypothesis can be constructed as,

$$
\begin{gathered}
F=\frac{\int \sum_{k=1}^{2} n_{k}\left(\widehat{\mu_{k}}(t)-\widehat{\mu}(t)\right)^{2} d t /(2-1)}{\int \sum_{k=1}^{2} \sum_{i=1}^{\mathrm{n}_{\mathrm{k}}}\left(g_{i k}(t)-\widehat{\mu_{k}}(t)\right)^{2} d t /(n-2),} \\
\text { where } \widehat{\mu_{k}}(t)=\sum_{i=1}^{n_{k}} g_{i k}(t) n_{k}^{-1} \text { and } \widehat{\mu}(t)=\sum_{k=1}^{2} \mathrm{n}_{\mathrm{k}} \widehat{\mu_{k}}(t) \mathrm{n}^{-1}
\end{gathered}
$$

\section{Simulation}

Simulations were conducted to compare the performance of the 3 methods using a simulation model specified here as well as on the Genetic Analysis Workshop 19 (GAW19) simulated phenotype data. First, we selected a subset of 142 unrelated individuals from the GAW19 family-based sequencing data. For each replicate, we randomly chose a $30-\mathrm{kb}$ segment from the $1.4 \mathrm{Mb}$ region (chromosome 3: 33100124 to 34539295$)$. From each segment, we randomly selected a specified proportion of SNVs (between 1 and $50 \%$ as given in Tables 1 and 2) as disease-associated SNVs. A logistic regression model was then applied to the selected SNVs to simulate a binary phenotype. In the simulation, we considered 2 types of effects, bidirectional effects and unidirectional effects, by randomly generating the regression coefficients from $\mathrm{N}(0$, $1)$ and $N(2,1)$, respectively. One thousand replicates were simulated for each scenario for power and type I error estimation. For FANOVA, we used the penalized cubic Bsplines to determine the smoothness of the functions. The smoothing parameter was determined by using the generalized cross validation.

The above simulations only evaluated 1 genetic region. To investigate the performance of the 3 methods on regions with different genetic structures, we also applied them to the subset of 142 unrelated samples from the GAW19 family-based simulated data, of which 24 samples are cases. This data consists of 294 genes, including THRA and RELB, which were simulated to be associated with the hypertension phenotypes. For the association analysis, hypertension (HTN1) from the first simulation out of the 200 simulations was used.

\section{Results}

Type I error rates of the 3 tests are well controlled at the 0.05 level (0.046 for BT, 0.044 for BTCOV, and 0.047 for FANOVA). As we observe from Table 1, power of the 3 tests increases as we increase the proportion of

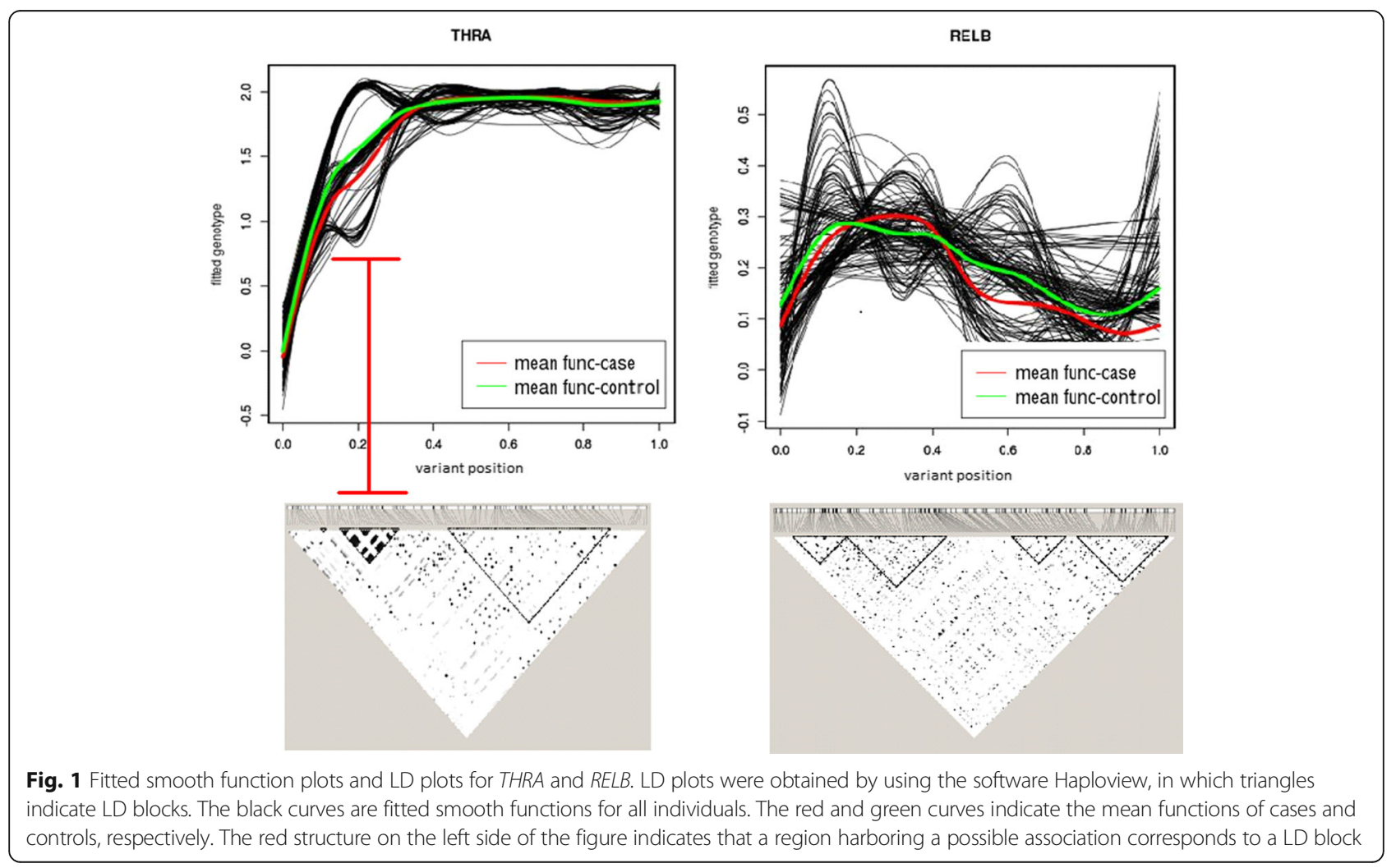


disease-associated variants. Overall, FANOVA has better or comparable performance to BT and BTCOV, while BTCOV obtains similar power to BT. The same conclusion also holds when the effects are bidirectional (see Table 2). We also observe that the power of the 3 tests is slightly lower in the case of bidirectional effects than in the case of unidirectional effects.

Table 3 summarizes the top 10 genes with the smallest $p$ values from the association analysis. Consistent with the result from simulations, we find that in general FANOVA attains smaller $p$ values, while the $p$ values of $\mathrm{BT}$ and BTCOV were comparable.

\section{Discussion}

Through this study, we find that overall BT and BTCOV have comparable performance. However, for 1 gene, THRA, BTCOV attains a lower $p$ value than the other 2 tests. In the follow-up analysis, we observe a small LD block in this gene (Fig. 1). The plot of the fitted genotype curves reveal the association happens to lie in that LD block. Therefore, BTCOV, which models the LD pattern, outperforms the other 2 tests. The plot also shows that FANOVA is able to capture the LD block. Nevertheless, the effects in the LD block are largely unidirectional, which is in favor of burden tests.

Variants may have more complex structure than pairwise LD. Hence, in most cases we find that FANOVA has comparable or better performance than the other 2 tests. For example, FANOVA attained a lower $p$ value than the other 2 tests in the analysis of $R E L B$. From Fig. 1, we observe that FANOVA not only captures the LD structure but also bidirectional effects, which are indicated by the crossing of the curves for cases and controls.

\section{Conclusions}

Our observations indicate that the performance of tests depends on the underlying genetic structure; hence, ignoring LD in the association analysis may not be ideal. It is advisable to use function-based approaches to explore and model the genetic structure. As illustrated by Fig. 1, the plot of the fitted functional curves provides a great way to explore the genetic structure. The diseaseassociated regions can also be visualized in this plot. If the underlying genetic structure tends to be complex (eg, having multiple LD blocks with different effects), it is also advisable to use function-based approaches, such as FANOVA, to adequately model the sequencing data.

\section{Acknowledgements}

The Genetic Analysis Workshop is supported by NIH grant R01 GM031575. The authors wish to thank the editor and two anonymous referees for their helpful comments that improve the manuscript.

\section{Declarations}

This article has been published as part of BMC Proceedings Volume 10 Supplement 7, 2016: Genetic Analysis Workshop 19: Sequence, Blood Pressure and Expression Data. Summary articles. The full contents of the supplement are available online at http://bmcproc.biomedcentral.com/articles/supplements/ volume-10-supplement-7. Publication of the proceedings of Genetic Analysis Workshop 19 was supported by National Institutes of Health grant R01 GM031575.

\section{Authors' contributions}

SJ conducted the data analysis and drafted the manuscript. OAV and XT helped set up the R code and prepare the simulation data for analysis. QL conceived of the study and helped finalize the manuscript. All 4 authors read and approved the final manuscript.

\section{Competing interests}

The authors declare they have no competing interests.

\section{Author details}

${ }^{1}$ Department of Statistics, Michigan State University, East Lansing, MI 48824, USA. ${ }^{2}$ Department of Epidemiology and Biostatistics, Michigan State University, East Lansing, Ml 48824, USA.

Published: 18 October 2016

\section{References}

1. Cirulli ET, Goldstein DB. Uncovering the roles of rare variants in common disease through whole-genome sequencing. Nat Rev Genet. 2010;11(6):415-25.

2. Lee S, Abecasis GR, Boehnke M, Lin X. Rare-variant association analysis: study designs and statistical tests. Am J Hum Genet. 2014;95(1):5-23.

3. Madsen $\mathrm{BE}$, Browning SR. A groupwise association test for rare mutations using a weighted sum statistic. PLoS Genet. 2009;5(2):e1000384.

4. Schaid DJ, McDonnell SK, Sinnwell JP, Thibodeau SN. Multiple genetic variant association testing by collapsing and kernel methods with pedigree or population structured data. Genet Epidemiol. 2013:37(5):409-18.

5. Vsevolozhskaya OA, Zaykin DV, Greenwood MC, Wei C, Lu Q. Functional analysis of variance for association studies. PLoS One. 2014:9(9):e105074.

6. Ramsay JO, Silverman BW. Functional data analysis. 2nd ed. New York: Springer; 2005.
Submit your next manuscript to BioMed Central and we will help you at every step:

- We accept pre-submission inquiries

- Our selector tool helps you to find the most relevant journal

- We provide round the clock customer support

- Convenient online submission

- Thorough peer review

- Inclusion in PubMed and all major indexing services

- Maximum visibility for your research

Submit your manuscript at www.biomedcentral.com/submit
Biomed Central 